\title{
Large spectral tuning of a water-glycerol microdroplet by a focused laser: characterization and modeling
}

\author{
A. Kiraz, ${ }^{a}$ Y. Karadağ $\breve{g}^{a}$ and M. Muradoğlu ${ }^{b}$
}

\author{
Received 23rd May 2008, Accepted 25th July 2008 \\ First published as an Advance Article on the web 22nd September 2008 \\ DOI: $10.1039 / \mathrm{b808772e}$
}

\begin{abstract}
Large spectral tuning of a water-glycerol microdroplet standing on a superhydrophobic surface by local heating with a focused infrared laser is studied both experimentally by optical spectroscopy and computationally using a lumped system formulation of the mass and heat transfer between the microdroplet and the chamber. The effects of optical scattering force, chamber humidity, size of microdroplet and laser power on the tuning mechanism are examined. The reversibility of the tuning mechanism is also studied. In spite of its negligibly small volatility compared to that of water, irreversibility is found to be mainly caused by evaporation of glycerol. It is also found that reversibility increases dramatically with the relative water and glycerol humidities, and spectral tuning can be made almost fully reversible when the chamber is saturated with glycerol vapor and the relative water humidity approaches unity. Some hysteresis effects are observed, especially in large microdroplets, and this behavior is attributed to the whisperinggallery mode resonances in laser absorption. The time response of the tuning mechanism is also analyzed both experimentally and computationally. The technique presented can find applications in optical communication systems, and can be used in fundamental studies in cavity quantum electrodynamics and in characterizing liquid aerosols on a surface.
\end{abstract}

\section{Introduction}

Largely tunable optical microcavities are important for applications in optical communication systems and fundamental studies in cavity quantum electrodynamics. ${ }^{1}$ Liquid microdroplets are attractive systems for such studies because of their easily deformable nature. We have previously demonstrated the spectral tuning of water microdroplets standing on a superhydrophobic surface by controlling the evaporation/condensation kinetics in a humidity chamber. ${ }^{2}$ Our demonstration relied on changing the temperature profile within the humidity chamber, which did not allow for local control of individual microdroplets. This resulted in a slow time response.

Evaporation/condensation kinetics of liquid microdroplets become much richer in the case of binary microdroplets which are constituted of a mixture of highly nonvolatile and volatile components. In particular, water-glycerol mixtures have been studied in detail as a model binary system. The evaporation and growth of water-glycerol microdroplets have been extensively examined in humidity-controlled environments by Ray and coworkers. ${ }^{3-5}$ In these studies, a single water-glycerol microdroplet is levitated electrodynamically and the size change controlled by the humidity of the chamber is measured highly accurately with Mie scattering experiments.

\footnotetext{
${ }^{a}$ Department of Physics, Koc University, Rumelifeneri Yolu, 34450 Sariyer Istanbul, Turkey.E-mail: akiraz@ku.edu.tr;

Fax: + 90 (0)212 3381559; Tel: + 90 (0) 2123381701

${ }^{b}$ Department of Mechanical Engineering, Koc University,

Rumelifeneri Yolu, 34450 Sariyer Istanbul, Turkey
}

In this article we characterize and model the spectral tuning of water-glycerol microdroplets standing on a superhydrophobic surface as a result of local heating induced by the absorption of a focused infrared laser. Under constant infrared laser illumination, individual water-glycerol microdroplets attain a temperature that is higher than the temperature of the humidity chamber. Being warmer than the surroundings, water in the microdroplet evaporates quickly and the microdroplet almost reaches an equilibrium at a new state with a smaller mole fraction of water; hence its size is reduced. When the infrared laser is switched off, the temperature of the microdroplet decreases and thus its size grows by water adsorption until a thermodynamic equilibrium is reached. At the end of this process, the microdroplet almost fully recovers its original size if the chamber is saturated with glycerol vapor. The reversibility of the present tuning mechanism increases dramatically with increasing relative water humidity in the chamber (i.e. for relative water humidities greater than $90 \%$ ).

In addition to its potential applications in optical communication systems and cavity quantum electrodynamics, the large spectral tuning method described in this article can prove to be important in characterizing liquid aerosols on a surface. For example, this method can be used for direct and noninvasive characterization of the thermodynamic properties and evaporation/condensation kinetics of simple and multi-component liquid aerosols. ${ }^{6,7}$

Our first results were presented in ref. 8 and 9. In ref. 8 the laser-induced size change of the microdroplet was mistakenly interpreted to be due to the optical scattering force. This interpretation error was then corrected in ref. 9. In this article we first show in section II that, within the range of laser 
powers used in our experiments, deformation due to the optical scattering force is much smaller than the resolution of the experimental setup, and is negligible compared to the size change caused by the evaporation and growth of the microdroplets due to laser-induced heating.

For over thirty years, optical spectroscopy experiments have been performed on liquid microdroplets ${ }^{10}$ flying in air or suspended using various position-stabilization techniques ${ }^{11}$ such as optical levitation, ${ }^{12,13}$ electrodynamic levitation ${ }^{3,4,14}$ and optical tweezing. ${ }^{15}$ In the experiments reported in this article we use a superhydrophobic surface for position stabilization. In addition to preserving the sphericity of the microdroplets, the use of the superhydrophobic surface improves the robustness of the experiments against external disturbances.

The article is organized as follows. In section II we calculate the deformation of a microdroplet due to the optical scattering force applied by a focused laser beam, and demonstrate that it cannot be the main mechanism responsible for the observed large spectral tuning. In section III we develop a rate equation model describing the evaporation and growth of a water-glycerol microdroplet that is locally heated by an infrared laser focused at the microdroplet's center. The localized approximation of the generalized Lorenz-Mie theory is used to calculate the absorption of the focused laser beam by the microdroplet. In section IV we describe the superhydrophobic surface preparation and experimental setup. In section $\mathrm{V}$ we present the results of the experiments performed under three relative humidity conditions, and show that they are in good qualitative agreement with the computational results obtained from the rate equation model described in section III. Conclusions are drawn in section VI.

\section{Negligible deformation due to the optical scattering force}

We first present a simple model to show that the large spectral tuning of water-glycerol microdroplets observed by Kiraz et al. ${ }^{8}$ cannot be due to the optical scattering force and the deformation caused by the optical scattering force is much smaller than the spectral resolution of the experimental setup for the range of laser powers used in the experiments. We assume that the focused laser beam propagates in the upward vertical direction inducing the optical scattering force $F_{\text {scat }}$, and the microdroplet takes a prolate spheroid shape with the minor axes of $r_{\mathrm{h}}$ and $r_{\mathrm{v}}$ in the horizontal and vertical directions, respectively, as sketched in
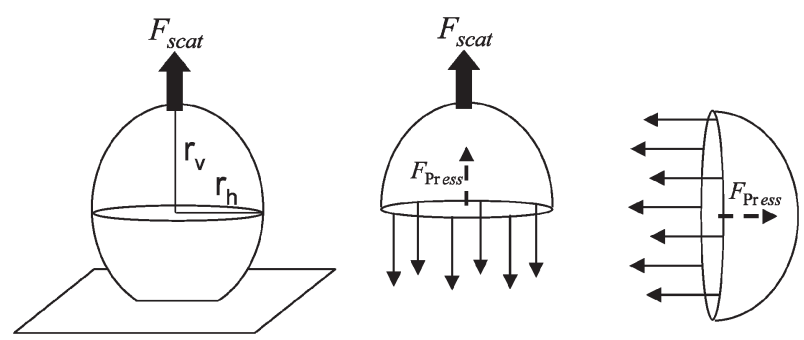

Fig. 1 Illustrations of deformation caused by the optical scattering force (left) and force balance in vertical (middle) and horizontal (right) directions.
Fig. 1. The microdroplet is assumed to be in an equlibrium, i.e. it takes a spherical shape in the absence of $F_{\text {scat }}$. Under these conditions, the force balance in the vertical direction yields

$$
F_{\text {scat }}=2 \pi r_{\mathrm{h}} \sigma_{\text {lv }}-\pi r_{\mathrm{h}}^{2} \Delta P,
$$

where $\sigma_{1 \mathrm{v}}$ is the surface tension and $\Delta P=P_{\text {in }}-P_{\text {out }}$ is the Laplace pressure defined as the difference between pressures inside and outside of the microdroplet. Ignoring effects of the substrate, the Laplace pressure can be obtained from the force balance in the horizontal direction as

$$
\Delta P=\frac{2 \sigma_{\mathrm{lv}}}{r_{\mathrm{v}}}
$$

Substituting eqn (2.2) into eqn (2.1) yields

$$
F_{\text {scat }}=2 \pi r_{\mathrm{h}} \sigma_{\mathrm{lv}}\left(1-\frac{r_{\mathrm{h}}}{r_{\mathrm{v}}}\right) \text {. }
$$

In the experiments by Kiraz et al. ${ }^{8} r_{\mathrm{h}}$ and $\sigma_{1 \mathrm{v}}$ are between $3.3-4.5 \mu \mathrm{m}$ and between $63 \times 10^{-3} \mathrm{~N} \mathrm{~m}^{-1}$ (glycerol) and $72 \times 10^{-3} \mathrm{~N} \mathrm{~m}^{-1}$ (water) respectively. Using the standard method presented in ref. 16, the scattering force is predicted to be $F_{\text {scat }} \sim 1-2 \mathrm{pN}$ for a microdroplet illuminated by a focused infrared laser beam of $5 \mathrm{~mW}$ incident power. Considering a microdroplet radius of $4 \mu \mathrm{m}$, the spectral shift is predicted to be $\Delta \lambda \approx 0.0004-0.0008 \mathrm{~nm}$ using the asymptotically linear relationship between the spectral position of the whisperinggallery modes (WGMs) and the normalized equatorial radius $\left(\Delta \lambda=\lambda \Delta r_{\mathrm{h}} / r_{\mathrm{h}}\right){ }^{17,18}$ This spectral shift is much smaller than the $0.17 \mathrm{~nm}$ spectral resolution of the experimental setup. ${ }^{8} \mathrm{We}$ thus conclude that the deformation due to the optical scattering force cannot be the main mechanism for the spectral shifts observed in the experiments of Kiraz et al. ${ }^{8}$ Note that this theoretical result is consistent with the recent experimental observations reported by Kiraz et al. ${ }^{9}$

\section{Theory}

A lumped equation model is presented in this section for the evaporation and growth of a water-glycerol microdroplet that is locally heated by an infrared laser beam focused on the microdroplet's center. Absorption of the focused laser beam by the microdroplet is calculated using the localized approximation of the generalized Lorenz-Mie theory. ${ }^{19-21}$ The theoretical predictions are compared with the experimental results in the next section.

\section{A. Rate equation model}

Following Ray et al., ${ }^{3,4}$ a simple lumped system formulation is used for mass and heat transfer between the microdroplet and the chamber. In this model, we assume that the composition and temperature distributions remain sufficiently uniform within the microdroplet for all times. This is a good approximation when the heat and mass transfer within the microdroplet is much more effective than the mass and heat transfer across a microdroplet interface, i.e. the Biot number is much smaller than unity. In addition, we assume that heat and mass transfer occur solely due to molecular diffusion in a quasistationary state, i.e. the evaporation and growth rates are sufficiently slow after a short transient period. Under these 
assumptions, the number of moles of component A (water) $N_{\mathrm{A}}$ and B (glycerol) $N_{\mathrm{B}}$ in the microdroplet of radius $a$ evolve by

$$
\begin{aligned}
\frac{\mathrm{d} N_{\mathrm{A}}}{\mathrm{d} t} & =-4 \pi a f_{\mathrm{PB}} D_{\mathrm{A}} \frac{P_{\mathrm{A}}^{0}\left(T_{\infty}\right)}{R T_{\infty}}\left(\gamma_{\mathrm{A}} x_{\mathrm{A}} \phi_{\mathrm{A}}-S_{\mathrm{A}}\right), \\
\frac{\mathrm{d} N_{\mathrm{B}}}{\mathrm{d} t} & =-4 \pi a f_{\mathrm{PB}} D_{\mathrm{B}} \frac{P_{\mathrm{B}}^{0}\left(T_{\infty}\right)}{R T_{\infty}}\left(\gamma_{\mathrm{B}} x_{\mathrm{B}} \phi_{\mathrm{B}}-S_{\mathrm{B}}\right),
\end{aligned}
$$

where

$$
\phi_{\mathrm{A}}=\left(\frac{T_{\infty}}{T_{\mathrm{d}}}\right) \frac{P_{\mathrm{A}}^{0}\left(T_{\mathrm{d}}\right)}{P_{\mathrm{A}}^{0}\left(T_{\infty}\right)} ; \phi_{\mathrm{B}}=\left(\frac{T_{\infty}}{T_{\mathrm{d}}}\right) \frac{P_{\mathrm{B}}^{0}\left(T_{\mathrm{d}}\right)}{P_{\mathrm{B}}^{0}\left(T_{\infty}\right)} .
$$

In eqns (3.4)-(3.6), the subscripts $\mathrm{A}$ and $\mathrm{B}$ denote properties of the components $\mathrm{A}$ and $\mathrm{B}, D_{\mathrm{A}, \mathrm{B}}$ is the molecular diffusivity in the gas mixture, $\gamma_{\mathrm{A}, \mathrm{B}}$ is the activity coefficient, $x_{\mathrm{A}, \mathrm{B}}$ is the mole fraction, $S_{\mathrm{A}, \mathrm{B}}$ is the relative humidity in the chamber, $R$ is the universal gas constant, $P_{\mathrm{A}, \mathrm{B}}^{0}(T)$ is the vapor pressure at temperature $T$, and $T_{\mathrm{d}}$ and $T_{\infty}$ are the temperatures at the microdroplet interface and in the chamber far from the microdroplet, respectively. The correction factor $f_{\mathrm{PB}}$ is introduced to account for the effects of the substrate on the mass transfer and is specified by Picknett and Bexon ${ }^{22,23}$ equation:

$f_{\mathrm{PB}}(\theta)=\frac{1}{2} \begin{cases}0.6366 \theta+0.09591 \theta^{2}-0.06144 \theta^{3} & 0^{\circ} \leq \theta<10^{\circ} \\ 0.000008957+0.6333 \theta+0.116 \theta^{2} & \\ -0.08878 \theta^{3}+0.01033 \theta^{4} & 10^{\circ} \leq \theta<180^{\circ},\end{cases}$

where the contact angle $\theta$ is in radians. In the present study, we take $\theta=160^{\circ}$ based on the experimental results obtained for millimetre-size droplets sitting on the superhydrophobic surface used here. Similarly, the temperature of the microdroplet evolves by

$$
\begin{aligned}
\frac{\mathrm{d} T_{\mathrm{d}}}{\mathrm{d} t}= & \frac{\Delta H_{\mathrm{vap}, \mathrm{A}}}{N C_{\mathrm{PL}}} \frac{\mathrm{d} N_{\mathrm{A}}}{\mathrm{d} t}+\frac{\Delta H_{\mathrm{vap}, \mathrm{B}}}{N C_{\mathrm{PL}}} \frac{\mathrm{d} N_{\mathrm{B}}}{\mathrm{d} t} \\
& -\frac{3 k_{\mathrm{eff}} V_{\mathrm{m}}}{a^{2} C_{\mathrm{PL}}}\left(T_{\mathrm{d}}-T_{\infty}\right)+\frac{\tilde{Q}_{\mathrm{abs}} P_{\text {inc }}}{N C_{\mathrm{PL}}},
\end{aligned}
$$

where $V_{\mathrm{m}}, N$ and $C_{\mathrm{PL}}$ are the molar specific volume, the total number of moles and molar heat capacity of the microdroplet mixture, $\Delta H_{\mathrm{vap}, \mathrm{A}, \mathrm{B}}$ is the enthalpy of evaporation and $k_{\mathrm{eff}}$ is the effective heat conductivity taken here as a simple area average of the heat conductivities of the air $\left(k_{\mathrm{a}}\right)$ and the substrate $\left(k_{\mathrm{s}}\right)$, i.e. $k_{\mathrm{eff}}=f_{\mathrm{a}} k_{\mathrm{a}} A_{\mathrm{a}}+\left(1-f_{\mathrm{a}}\right) k_{\mathrm{s}}$ where $f_{\mathrm{a}}=A_{\mathrm{a}} / A_{\mathrm{d}}$ is the ratio of the surface area of the microdroplet that is in contact with the air and the total surface area of the microdroplet. The last term in eqn (3.8) represents the heating of the microdroplet by the focused infrared laser beam. $\tilde{Q}_{\mathrm{abs}}$ is the ratio of the total power absorbed by the microdroplet to the total power of the incident beam $\left(P_{\text {inc }}\right)$.

The activity coefficient of water $\left(\gamma_{\mathrm{A}}\right)$ is computed from the van Laar equation

$$
\ln \gamma_{\mathrm{A}}=\frac{c_{1}}{\left(1+\frac{c_{1} x_{\mathrm{A}}}{c_{2} 1-x_{\mathrm{A}}}\right)^{2}}
$$

where the parameters $c_{1}=-0.3049$ and $c_{2}=-0.8551$ are obtained from the best fit to the experimental data by
Tu and Ray. ${ }^{3}$ The activity coefficient of glycerol $\left(x_{\mathrm{B}}\right)$ is then obtained from the Gibbs-Duhem equation: ${ }^{4}$

$$
\ln \gamma_{\mathrm{B}}=\frac{c_{2}}{c_{1}}\left(2 \sqrt{c_{1} \ln \gamma_{\mathrm{A}}}+\ln \gamma_{\mathrm{A}}+c_{1}\right)
$$

Descriptions of the variables are summarized in Table 1 and the values of the constants used in the calculations are given in Table 2. Eqn (3.4), (3.5) and (3.9) are integrated in time using MATLAB $^{\circledR 24}$ stiff ODE solver ODE15s. Detailed computational results are presented in section $\mathrm{V}$ and compared with the experimental data.

At this stage it is instructive to analyze the behavior of the rate eqn (3.4) and (3.8) assuming that glycerol is ideally nonvolatile. Under a constant laser illumination, the steadystate value of $T_{\mathrm{d}}$ is larger than $T_{\infty}$ by $\tilde{Q}_{\mathrm{abs}} P_{\text {inc }} a^{2} / 3 k_{\mathrm{g}} V_{\mathrm{m}} N$. For this value of $T_{\mathrm{d}}, x_{\mathrm{A}}$ is then obtained from the steady state relation $\gamma_{\mathrm{A}} x_{\mathrm{A}} \phi_{\mathrm{A}}=S_{\mathrm{A}}$. The steady-state solution of $x_{\mathrm{A}}$ is plotted in Fig. $2 \mathrm{a}$ as a function of $S_{\mathrm{A}}$ for various values of $T_{\mathrm{d}}$ ranging between 299.55 and $304.55 \mathrm{~K}$, and assuming $T_{\infty}=$ $299.55 \mathrm{~K}$. This Figure shows that $x_{\mathrm{A}}$ decreases with increasing $T_{\mathrm{d}}$ at a fixed value of $S_{\mathrm{A}}$ and the reduction in $x_{\mathrm{A}}$ becomes more pronounced as the relative humidity of water in the chamber increases for a unit increment in $T_{\mathrm{d}}$. It is also interesting to

\begin{tabular}{|c|c|}
\hline Constants and variables & Explanation \\
\hline$a$ & Microdroplet radius \\
\hline$x_{\mathrm{A}}$ & Mole fraction of water \\
\hline$x_{\mathrm{B}}=\left(1-x_{\mathrm{A}}\right)$ & Mole fraction of glycerol \\
\hline$V_{\mathrm{A}}$ & Molar volume of water \\
\hline$V_{\mathrm{B}}$ & Molar volume of glycerol \\
\hline$V_{\mathrm{m}}=x_{\mathrm{A}} V_{\mathrm{A}}+x_{\mathrm{B}} V_{\mathrm{B}}$ & Molar volume of the microdroplet \\
\hline & Gas constant \\
\hline$\Delta H_{\mathrm{vap}, \mathrm{A}}$ & Latent heat of vaporization of water \\
\hline$\Delta H_{\mathrm{vap}, \mathrm{B}}$ & Latent heat of vaporization of glycerol \\
\hline$C_{\mathrm{PA}}$ & Molar heat capacity of water \\
\hline$C_{\mathrm{PB}}$ & Molar heat capacity of glycerol \\
\hline$C_{\mathrm{PL}}=x_{\mathrm{A}} C_{\mathrm{PA}}+x_{\mathrm{B}} C_{\mathrm{PB}}$ & Molar heat capacity of the microdroplet \\
\hline$D_{\mathrm{A}}$ & $\begin{array}{l}\text { Gas-phase diffusion coefficient of water } \\
\text { in air }\end{array}$ \\
\hline$D_{\mathrm{B}}$ & $\begin{array}{l}\text { Gas-phase diffusion coefficient of glycerol } \\
\text { in air }\end{array}$ \\
\hline$k_{\mathrm{a}}$ & Thermal conductivity of air \\
\hline$k_{\mathrm{s}}$ & Thermal conductivity of the substrate \\
\hline$T_{\mathrm{d}}$ & Microdroplet temperature \\
\hline & Temperature of air in the chamber \\
\hline$P_{\mathrm{A}}^{0}(T)$ & Vapor pressure of water at temperature $T$ \\
\hline$P_{\mathrm{B}}^{0}(T)$ & $\begin{array}{l}\text { Vapor pressure of glycerol at temperature } \\
T\end{array}$ \\
\hline$S_{\mathrm{A}}$ & Saturation ratio of water in air \\
\hline$S_{\mathrm{B}}$ & Saturation ratio of glycerol in air \\
\hline$N_{\mathrm{A}}$ & $\begin{array}{l}\text { Number of moles of water in the } \\
\text { microdroplet }\end{array}$ \\
\hline$N_{\mathrm{B}}$ & $\begin{array}{l}\text { Number of moles of glycerol in the } \\
\text { microdroplet }\end{array}$ \\
\hline$N=x_{\mathrm{A}} N_{\mathrm{A}}+x_{\mathrm{B}} N_{\mathrm{B}}$ & Total number of moles in the microdroplet \\
\hline$n_{\mathrm{A}}$ & Refractive index of water at $1064 \mathrm{~nm}$ \\
\hline$n_{\mathrm{B}}$ & Refractive index of glycerol at $1064 \mathrm{~nm}$ \\
\hline & Wavelength of incident light \\
\hline$\alpha=2 \pi a / \lambda$ & Size parameter \\
\hline
\end{tabular}
observe that a small increase in droplet temperature $\left(T_{\mathrm{d}}\right)$ results in large reduction in microdroplet size at large relative water humidities in the chamber, e.g. $S_{\mathrm{A}}>0.9$, since the volatility of water is much larger than that of glycerol and the microdroplet contains very little amount of glycerol at large

Table 1 Nomenclature 
Table 2 Constants 5,27

\begin{tabular}{lll}
\hline Constant & Value & $\mathrm{Unit}$ \\
\hline$V_{\mathrm{A}}$ & $18.0153 \times 10^{-6}$ & $\mathrm{~m}^{3} \mathrm{~mol}^{-1}$ \\
$V_{\mathrm{B}}$ & $73.03 \times 10^{-6}$ & $\mathrm{~m}^{3} \mathrm{~mol}^{-1}$ \\
$R$ & 8.314472 & $\mathrm{~J} \mathrm{~mol}^{-1} \mathrm{~K}^{-1}$ \\
$\Delta H_{\text {vap,A }}$ & 40714.578 & $\mathrm{~J} \mathrm{~mol}^{-1}$ \\
$\Delta H_{\text {vap,B }}$ & 61000 & $\mathrm{~J} \mathrm{~mol}^{-1}$ \\
$C_{\mathrm{PA}}$ & 75.327 & $\mathrm{~J} \mathrm{~mol}^{-1} \mathrm{~K}^{-1}$ \\
$C_{\mathrm{PB}}$ & 221.193 & $\mathrm{~J} \mathrm{~mol}^{-1} \mathrm{~K}^{-1}$ \\
$D_{\mathrm{A}}$ & $24.2 \times 10^{-6}$ & $\mathrm{~m}^{2} \mathrm{~s}^{-1}$ \\
$D_{\mathrm{B}}$ & $8.7710 \times 10^{-6}$ & $\mathrm{~m}^{2} \mathrm{~s}^{-1}$ \\
$k_{\mathrm{a}}$ & 0.02620 & $\mathrm{~W} \mathrm{~m} \mathrm{~K}^{-1}$ \\
$k_{\mathrm{s}}$ & 1.14 & $\mathrm{~W} \mathrm{~m} \mathrm{~K}^{-1}$ \\
$n_{\mathrm{A}}$ & $1.33+1202.32 \times 10^{-9} \mathrm{i}$ & - \\
$n_{\mathrm{B}}$ & $1.47+1811.95 \times 10^{-9} \mathrm{i}$ & - \\
\hline
\end{tabular}
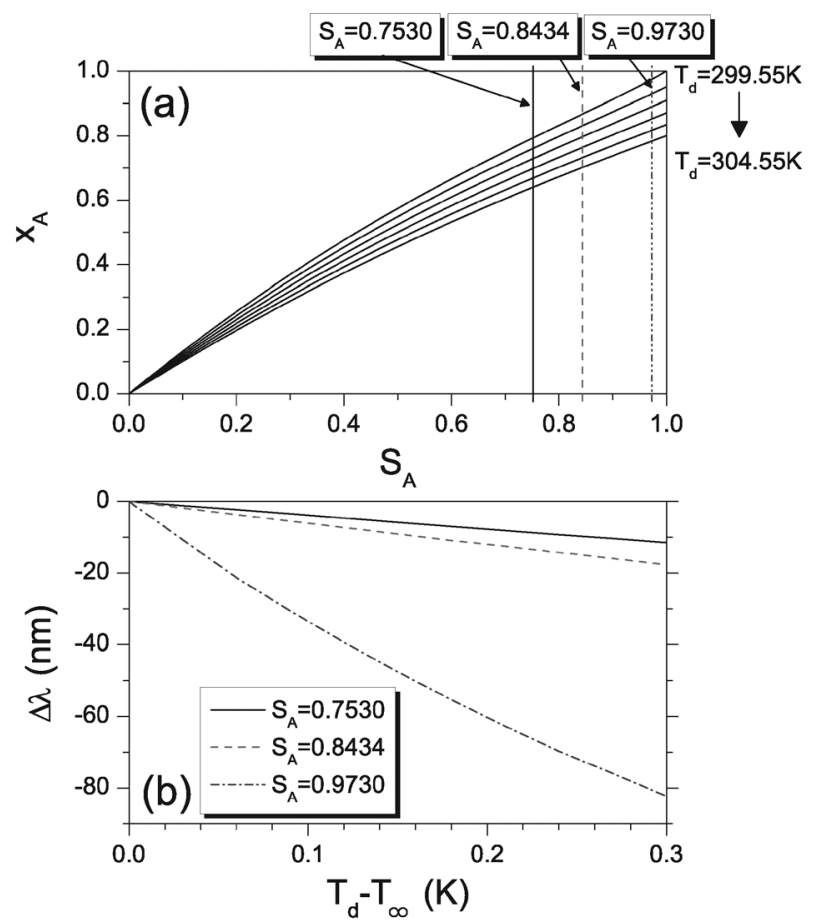

Fig. 2 Steady-state analysis assuming that glycerol is ideally nonvolatile. (a) Steady mole fraction of water as a function of relative humidity of water for various values of microdroplet temperature $\left(T_{\mathrm{d}}\right)$ assuming the temperature of the chamber $\left(T_{\infty}\right)$ to be $299.55 \mathrm{~K}$. Vertical lines indicate the relative humidities of water used in the experiments. (b) The spectral drift in the WGMs located at around $590 \mathrm{~nm}$ as a function of $T_{\mathrm{d}}-T_{\infty}$.

values of $S_{\mathrm{A}}$. This implies that a large relative water humidity in the chamber ensures a large tuning range and is the essence of the spectral tuning mechanism presented in this article. This is also verified in Fig. $2 b$ where the spectral shifts in the WGMs located at around $590 \mathrm{~nm}$ are plotted as a function of $T_{\mathrm{d}}-T_{\infty}$ for three different $S_{\mathrm{A}}$ values used in the experiments. The calculations in Fig. 2 b use the asymptotically linear relationship between the spectral position of the WGMs and the normalized equatorial radius, ${ }^{17,18}$ and they are valid for WGMs at around $590 \mathrm{~nm}$ irrespective of the size of the microdroplet. The range of temperature differences in this Figure corresponds to that caused by laser heating used in our experiments, as will be discussed in section V. Note that

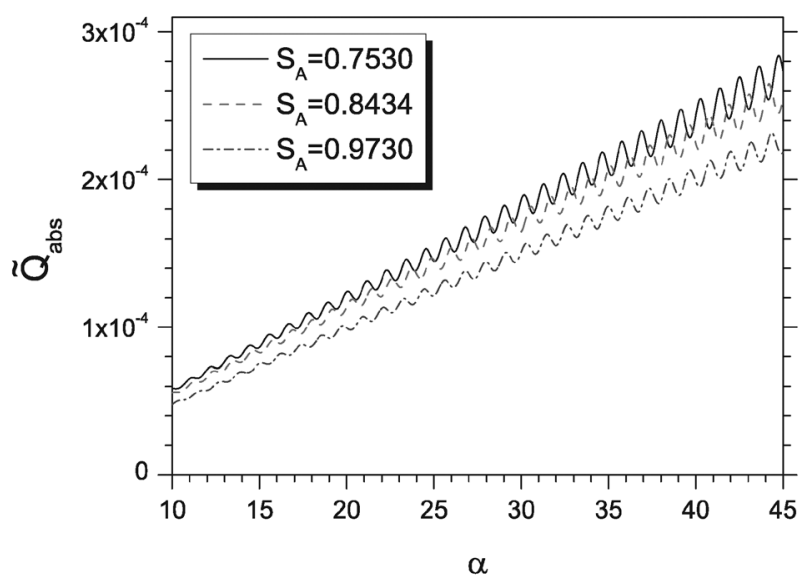

Fig. 3 Normalized absorption efficiency as a function of the structure factor $(\alpha=2 \pi a / \lambda)$ for the three different relative water humidities used in the experiments. A perfectly spherical microdroplet is assumed to be suspended in air, and the effects of the superhydrophobic substrate are ignored. Low quality oscillations are due to the Fabry-Perot resonace modes.

the results plotted in Fig. 2 represent a fully reversible tuning mechanism since glycerol is assumed to be ideally nonvolatile. When evaporation of the glycerol is also considered, the tuning mechanism becomes slightly irreversible. This will be dicussed in detail in section $\mathrm{V}$.

\section{B. Laser-induced heating}

Laser-induced heating is computed using the localized approximation of the generalized Lorenz-Mie theory applied to the absorption of a focused laser beam by a spherical microdroplet suspended in air. ${ }^{19-21}$ The calculations are based on the correction coefficients given in ref. 19 for on-axis illumination. The normalized absorption efficiency $\left(\tilde{Q}_{\mathrm{abs}}\right)$ is plotted in Fig. 3 as a function of the structure factor $(\alpha=2 \pi a / \lambda)$ for three relative water humidities used in the experiments. In these calculations, wavelength and focal waist are taken as $\lambda=1064 \mathrm{~nm}$ and $w_{0}=$ $1000 \mathrm{~nm}$. The refractive index of the microdroplet is calculated as: $n_{\mathrm{L}}=v_{\mathrm{A}} n_{\mathrm{A}}+v_{\mathrm{B}} n_{\mathrm{B}},{ }^{5}$ where $v_{\mathrm{A}}$ and $v_{\mathrm{B}}$ denote the volume fractions of water and glycerol respectively. The complex refractive indices of water and glycerol are taken as $n_{\mathrm{A}}=1.33+1202.32 \times 10^{-9} \mathrm{i}$ and $n_{\mathrm{B}}=1.47+1811.95 \times 10^{-9} \mathrm{i}$, assuming the absorption coefficients in ref. 25 . Due to on-axis illumination, the high quality WGMs are not observed in Fig. 3 and the low quality oscillations correspond to the Fabry-Perot resonance modes. ${ }^{26}$ As expected, for large values of $\alpha$, i.e. $\alpha>50$, the present calculations almost exactly match with the absorption of a dielectric slab with a thickness of $2 a$. Note that the omission of the superhydrophobic surface is a source of error in the present calculations. A simple three dielectric layer (glass/microdroplet/air) model indicates that the omission of substrate may result in up to $5 \%$ more laserinduced heating than the actual value.

\section{Surface preparation and experimental setup}

Superhydrophobic surfaces were prepared by spin coating $50 \mathrm{mg} \mathrm{ml} \mathrm{m}^{-1}$ ethanol dispersions of hydrophobically-coated silica nanoparticles (Evonik, Aeroxide ${ }^{\mathbb{R}}$ LE1) on cover glasses 
as reported previously. ${ }^{2,8}$ The average contact angle of millimetre-sized droplets was measured to be larger than $155^{\circ}$ on these surfaces. Water-glycerol microdroplets were generated with an ultrasonic nebulizer at room temperature and pressure using a solution with a 90:10 volume distribution of water:glycerol, containing $5 \mu \mathrm{M}$ rhodamine $\mathrm{B}$. Following microdroplet generation, water content in the microdroplets quickly evaporates, resulting in microdroplets with diameters ranging from a few up to $\sim 20 \mu \mathrm{m}$. A superhydrophobic surface containing the microdroplets is then placed in the humidity chamber having a controlled relative humidity. After waiting for the growth of the microdroplets to their equilibrium sizes for several hours, optical experiments are performed.

The humidity chamber and the optical setup used in the experiments are shown in Fig. 4. A saturated salt-water solution is kept in the humidity chamber in order to have a high and stable relative humidity. Saturated water solutions of three different salts are used in the experiments: (I) $\mathrm{NaCl}$, (II) $\mathrm{KCl}$, and (III) $\mathrm{K}_{2} \mathrm{SO}_{4}$. Calibrated relative humidities are $S_{\mathrm{A}}=0.7530,0.8434$ and 0.9730 for cases I, II and III respectively. $^{27}$

A home-made continuous wave (cw) infrared solid-state laser ( $\lambda=1064 \mathrm{~nm}$, maximum output power: $\sim 1 \mathrm{~W}$ ) is used for the local heating of microdroplets (Fig. 4). The infrared laser is transmitted through a $2 \times$ magnification telescope, a continuously variable neutral density filter, a neutral density filter used as a beam combiner, reflected by a dichroic mirror and focused to the center of individual microdroplets using a high numerical aperture microscope objective $(\mathrm{NA}=1.4$ oil, $60 \times$ ), in the inverted geometry. WGMs are observed by recording the rhodamine $\mathrm{B}$ emission spectra collected from individual micro-

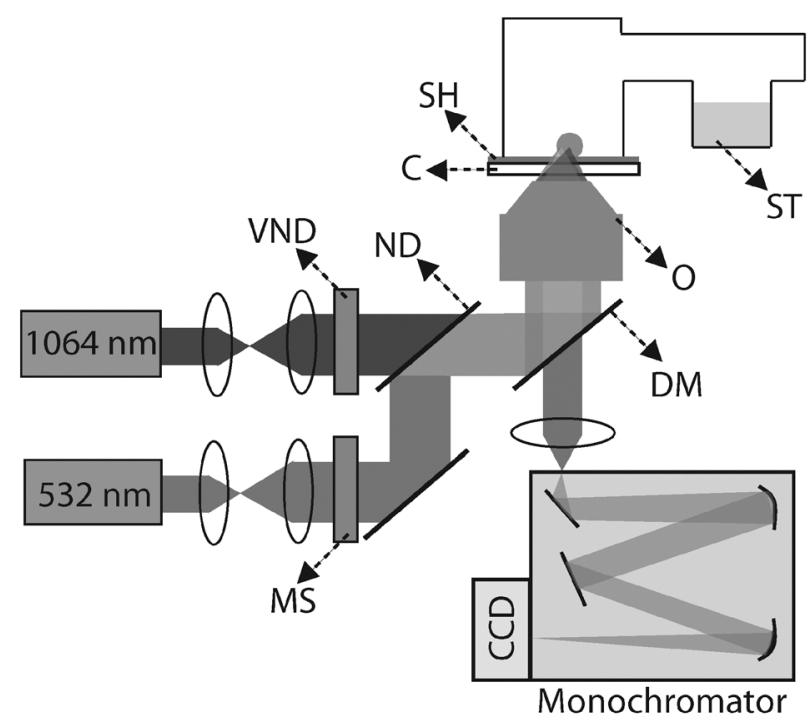

Fig. 4 Illustration of the experimental setup. VND: variable neutral density filter; ND: neutral density filter; MS: mechanical shutter; DM: dichroic mirror; O: microscope objective; C: cover glass; $\mathrm{SH}$ : superhydrophobic coating; ST: saturated salt-water solution. A continuous wave $(\mathrm{cw})$ infrared solid-state laser was used for local heating and a cw green solid-state laser used for the excitation of the rhodamine B molecules. The microdroplet is on the cover glass coated with hydrophobically-coated silica nanoparticles. droplets upon their excitation by a cw green solid-state laser $(\lambda=532 \mathrm{~nm})$. The green laser is transmitted through a $6 \times$ telescope, reflected by the neutral density filter used as the beam combiner and the dichroic mirror, and focused approximately on the rim of the microdroplets. Rhodamine B emission is collected with the same microscope objective, transmitted through the dichroic mirror and a $1.5 \times$ magnification element, dispersed by a $50 \mathrm{~cm}$ monochromator (600 grooves per $\mathrm{mm}$ grating), and detected by a CCD camera using an exposure time of $0.5 \mathrm{~s}$ with a spectral resolution of $0.17 \mathrm{~nm}$. A mechanical shutter is used to block the green laser during the unexposed periods, while the infrared laser is always illuminating the microdroplets at varying intensities controlled with the continuously variable neutral density filter. The mechanical shutter is used to block both the infrared and green lasers only for the experiments performed to resolve the time response of the spectral tuning mechanism (Fig. 9). Excitation powers at the focus of the microscope objective are $\sim 1 \mu \mathrm{W}$ for the green laser and between $0-5 \mathrm{~mW}$ for the infrared laser.

\section{Results and discussion}

We now present the experimental results together with the theoretical predictions. Fig. 5 shows exemplary emission spectra obtained from a $7.6 \mu \mathrm{m}$ diameter microdroplet during spectral tuning experiments performed under a relative water humidity of $S_{\mathrm{A}}=0.8434$. The incident laser power is first gradually increased from zero to the maximum power of

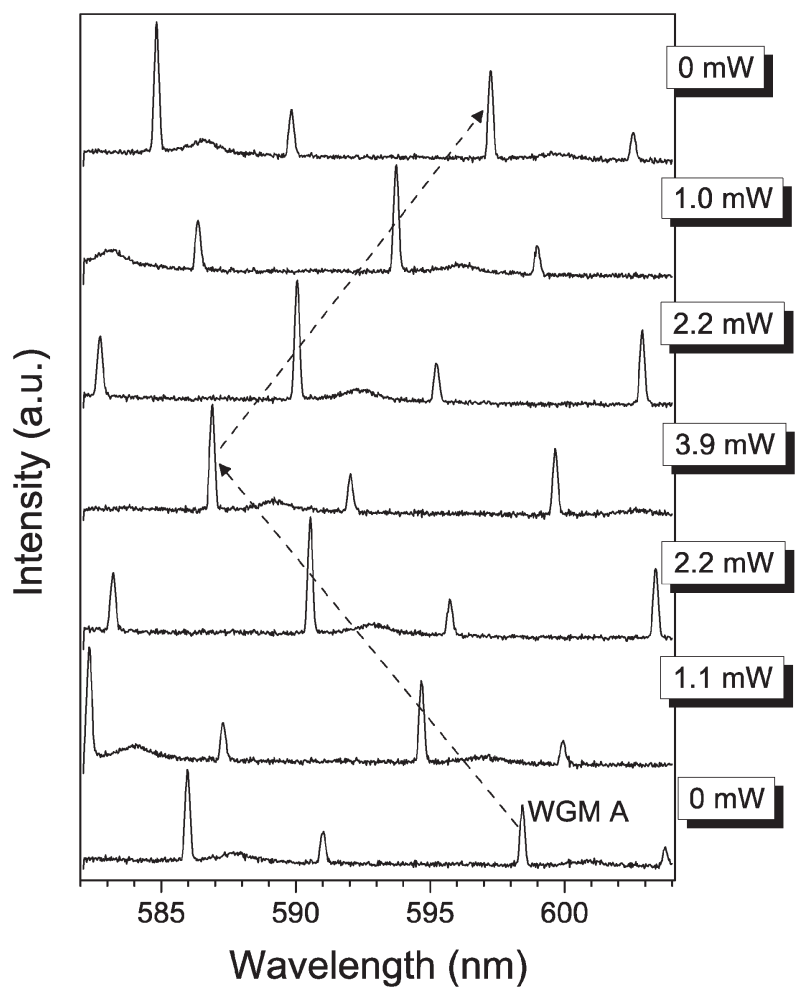

Fig. 5 Emission spectra recorded from a $7.6 \mu \mathrm{m}$ diameter microdroplet while the infrared laser power is first increased from and then decreased to zero. Arrows indicate the spectral drift direction of the high quality WGMs belonging to the same mode set. At the maximum infrared laser power of $3.9 \mathrm{~mW}$, total spectral drift is $11.5 \mathrm{~nm}$. 
$3.9 \mathrm{~mW}$ resulting in the blue shift of the WGMs. The incident laser power is then gradually decreased to zero resulting in the red shift of the WGMs. Arrows in this Figure indicate the spectral drift of a high quality WGM (WGM A) having a resolution-limited spectral width of $0.17 \mathrm{~nm}$. A total spectral drift of $11.5 \mathrm{~nm}$ is observed in this WGM at the maximum infrared laser power of $3.9 \mathrm{~mW}$. At the end of the experiment, WGM A is observed to be blue shifted by $1.2 \mathrm{~nm}$ compared to its spectral position at the beginning of the experiment, because of the evaporation of glycerol. The high quality lineshape function of the WGM A is well preserved during spectral tuning. This indicates that the sphericity and the contact angle of the microdroplet did not change considerably throughout the experiment. ${ }^{28}$

We next present in Fig. 6a the spectral drifts $(\Delta \lambda)$ observed in WGMs of various microdroplets as a function of incident laser power $\left(P_{\text {inc }}\right)$ for three relative water humidities of $S_{\mathrm{A}}=0.753,0.8434$ and 0.973 . Solid lines in Fig. 6a correspond to the measurements performed for different microdroplets with diameters ranging between $6.4-8.7,6.4-8.1$ and $7.0-11.1 \mu \mathrm{m}$ for $S_{\mathrm{A}}=0.7530,0.8434$ and 0.9730 respectively. Computational results assuming $7 \mu \mathrm{m}$ microdroplet diameter and $T_{\infty}=299.55 \mathrm{~K}$ are also shown in the Figure as dashed lines. For all cases a nearly linear relationship is observed between $\Delta \lambda$ and $P_{\text {inc }}$, which is in good qualitative agreement with the computational results. Besides, for a given relative water humidity and incident laser power, the experiments reveal similar spectral drifts for all microdroplet sizes. This is in agreement with both the analysis done in Fig. $2 b$ and spectral drift calculations made for microdroplets with diameters ranging between 6-12 $\mu \mathrm{m}$ (Fig. 6c). In Fig. 6a the predicted spectral drifts are smaller than the measured values in all three values of $S_{\mathrm{A}}$. In addition to simplifications made in a lumped system formulation, this difference is partly attributed to the omission of the superhydrophobic substrate in computing laser-induced heating. As mentioned before, a simple dielectric slab model indicates that the omission of the substrate may result in up to $5 \%$ more laser heating than the actual value.

The temperature increase in the microdroplet due to laser heating is not measured in the present study but it can be predicted from the lumped equation model. The predicted temperature rise in a $7 \mu \mathrm{m}$ diameter microdroplet due to laser heating is plotted in Fig. $6 \mathrm{~b}$ as a function of the incident laser power for three relative water humidities. The Figure shows that the temperature difference between the microdroplet and the chamber is slightly larger for smaller values of $S_{\mathrm{A}}$ and is less than $0.25 \mathrm{~K}$ for all the cases in the range of laser powers used in the experiments. It is interesting to see that this small temperature difference causes a significant spectral shift as seen in Fig. 6a. As presented in Fig. 6d, the computations performed for microdroplet diameters ranging between 6-12 $\mu \mathrm{m}$ for $S_{\mathrm{A}}=0.9730$ show that the temperature rise in the microdroplet is not considerably affected by its size for a given relative water humidity and incident laser power. This is in agreement with the steady-state solution for the
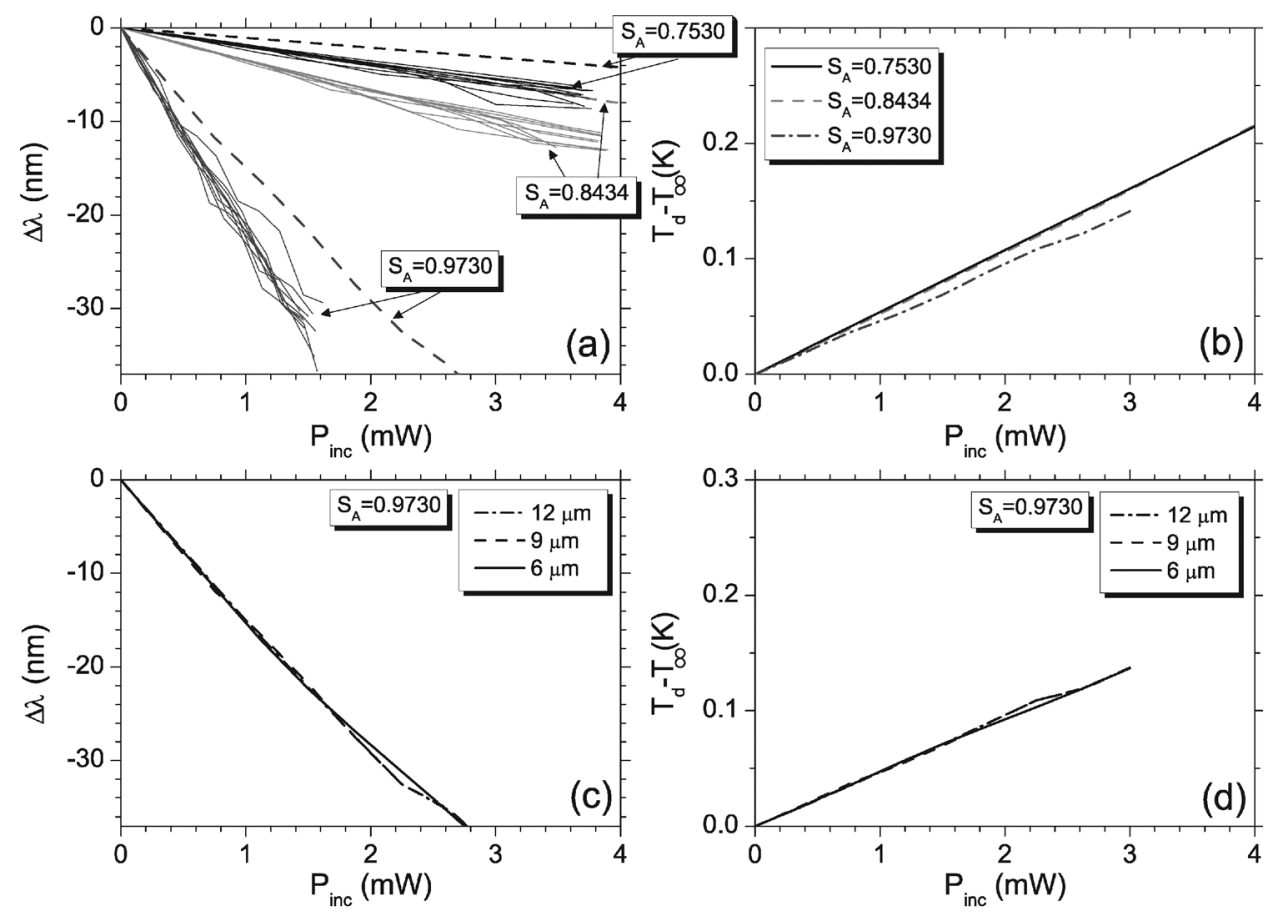

Fig. 6 (a) Computational (dashed lines) and experimental (solid lines) spectral drifts observed in WGMs at around $590 \mathrm{~nm}$ as a function of incident laser power for three different relative water humidities. Computational spectral drifts are calculated assuming $7 \mu \mathrm{m}$ microdroplet diameter. Experimental spectral drifts are recorded from different microdroplets with various sizes ranging between 6.4-8.7, 6.4-8.1 and 7.0-11.1 $\mu \mathrm{m}$ for $S_{\mathrm{A}}=0.7530,0.8434$ and 0.9730 respectively. (b) Computational results of temperature change of a $7 \mu \mathrm{m}$ diameter microdoplet as a function of incident laser power for three different relative water humidities. (c) and (d) Computational results showing the spectral drifts (c) and temperature changes (d) of 6,9 and $12 \mu \mathrm{m}$ diameter microdroplets as a function of incident laser power for $S_{\mathrm{A}}=0.9730$. The curves are hardly distinguishable in (c) and (d) showing that spectral drift and temperature change are not considerably affected by the microdroplet size. 
temperature rise in the microdroplet, i.e. $T_{\mathrm{d}}-T_{\infty}=$ $\tilde{Q}_{\text {abs }} P_{\text {inc }} a^{2} / 3 k_{\text {eff }} V_{\mathrm{m}} N$. In this equation $N$ is proportional to $a^{3}$, and $\tilde{Q}_{\text {abs }}$ has an almost linear relationship with $a$, hence the rise in the microdroplet temperature is essentially independent of $a$ to a good approximation.

We now examine the reversibility of the tuning mechanism. Solid lines in Fig. 7 show the spectral drifts observed from three microdroplets kept at constant relative water humidities while the laser power is gradually increased from zero to a peak value and is decreased again gradually from the peak value to zero. Microdroplet diameters are 6.4, 7.0 and $7.0 \mu \mathrm{m}$ for $S_{\mathrm{A}}=0.7530,0.8434$ and 0.9730 respectively. Dashed lines show the computational results obtained using these experimental parameters. In simulations we assume that the microdroplets are exposed to the infrared laser for $8 \mathrm{~s}$ at each laser power. This time interval corresponds to the average time elapsing between consecutive spectra recorded in our experiments. The first conclusion that can be drawn from Fig. 7 is that the spectral tuning process is not fully reversible, i.e. the microdroplet does not fully recover its initial size when the laser power is switched off. Another important observation is that the reversibility of spectral tuning is enhanced

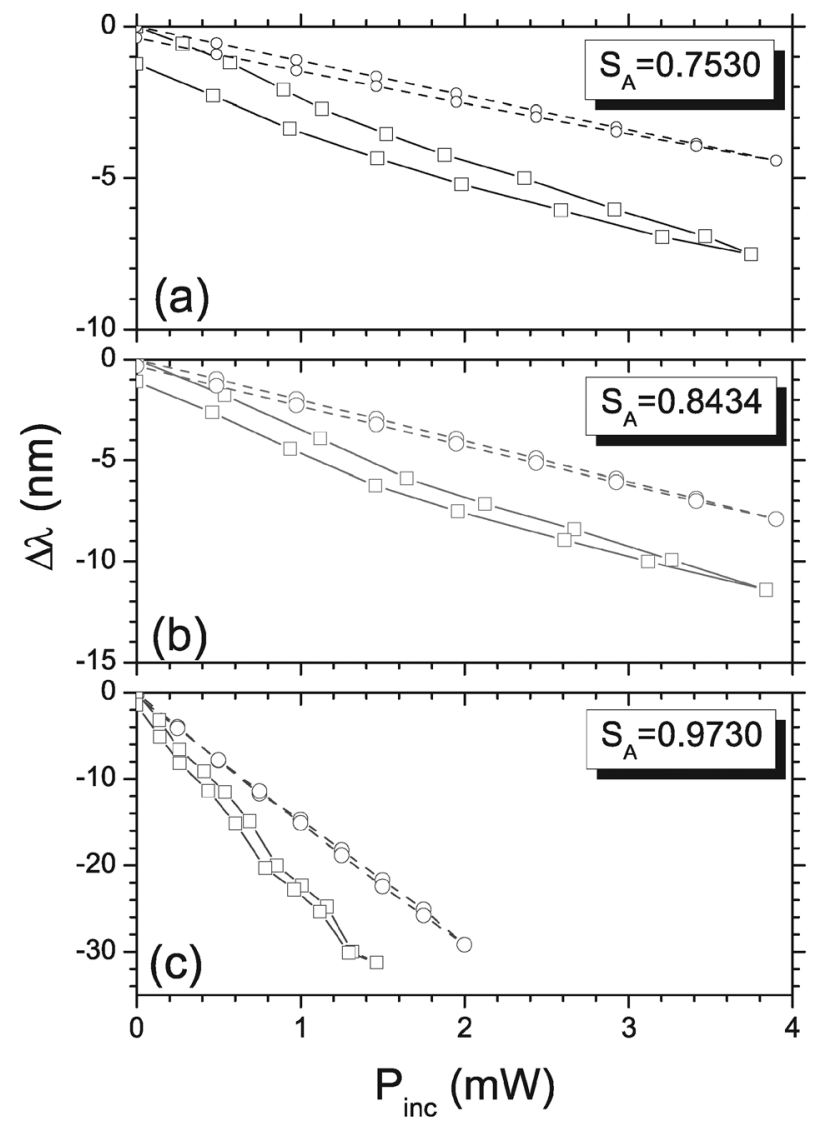

Fig. 7 The reversibility of spectral tuning for the relative water humidities of (a) $S_{\mathrm{A}}=0.7530$, (b) $S_{\mathrm{A}}=0.8434$ and (c) $S_{\mathrm{A}}=$ 0.9730. Microdroplet diameters are 6.4, 7.0 and $7.0 \mu \mathrm{m}$ in (a), (b) and (c) respectively. The solid lines with squares indicate the experimental data and dashed lines with circles indicate the computational results obtained by assuming that the chamber is initially saturated with glycerol vapor. significantly by increasing relative humidity of water in the chamber, which implies that the irreversibility is mainly caused by the evaporation of glycerol content of the microdroplet. From the solid lines in Fig. 7, spectral drifts of $-1.2,-1.1$ and $-1.4 \mathrm{~nm}$ are observed at the end of the spectral tuning cycles in which maximum drifts of $-7.5,-11.4$ and $-31.2 \mathrm{~nm}$ are observed for $S_{\mathrm{A}}=0.7530,0.8434$ and 0.9730 respectively. Hence for $S_{\mathrm{A}}=0.7530,0.8434$ and 0.9730 the spectral drift at the end of the tuning cycle is $16,9.6$ and $4.5 \%$ of the maximum spectral tuning observed within the cycle.

Although there is a qualitative agreement between the experimental and computational results, the predicted irreversibilities are much smaller than those observed in the experiments for all three values of $S_{\mathrm{A}}$. This difference can be explained by the fact that the simulations are performed by assuming that the relative humidity of glycerol in the chamber is in equilibrium conditions $\left(S_{\mathrm{B}}=S_{\mathrm{B} \text {,eq }}\right)$, i.e. air in the chamber is saturated with glycerol vapor. This means that the most of the glycerol that has evaporated during laser heating is regained by the microdroplet through condensation of glycerol vapor in the chamber when the laser is switched off. In order to verify this, computations are performed at $S_{\mathrm{A}}=0.8434$ for various values of $S_{\mathrm{B}}$, and the results are plotted in Fig. 8. The total spectral drifts of $-0.3,-1.0$ and $-1.6 \mathrm{~nm}$ are calculated at the end of the tuning cycles for $S_{\mathrm{B}}=S_{\mathrm{B}, \mathrm{eq}}, 0.9 S_{\mathrm{B}, \mathrm{eq}}$ and $0.8 S_{\mathrm{B}, \mathrm{eq}}$ respectively. Hence the reversibility is improved significantly as $S_{\mathrm{B}}$ increases toward its equilibrium value, i.e. $S_{\mathrm{B}}=0.0876$ in this case. Note that the equilibrium humidity of glycerol $\left(S_{\mathrm{B}, \text { eq }}\right)$ is computed from the conditions: $\gamma_{\mathrm{A}} x_{\mathrm{A}}=S_{\mathrm{A}}$ and $\gamma_{\mathrm{B}} x_{\mathrm{B}}=S_{\mathrm{B}, \mathrm{eq}}$ at $T_{\mathrm{d}}=T_{\infty}$.

Measurements are also performed to analyze the transient response of the spectral tuning mechanism. In these experiments the mechanical shutter is used to block both the infrared and green lasers, and the fluorescence spectra are recorded as the size of the microdroplet changes during varying time intervals in which shutter is opened. Transient measurements

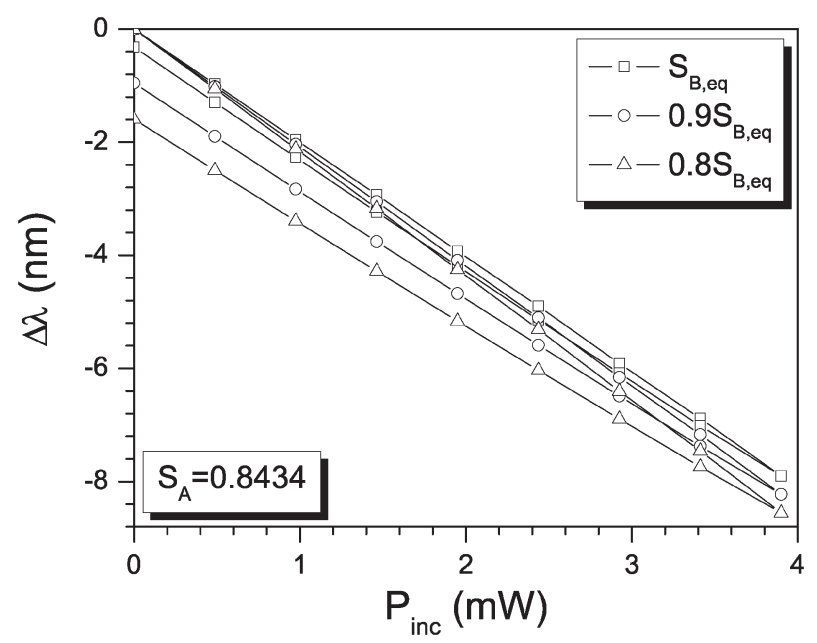

Fig. 8 The effect of the relative humidity of glycerol on the reversibility of spectral tuning. Computations are performed at $S_{\mathrm{A}}=0.8434$ for $S_{\mathrm{B}}=S_{\mathrm{B}, \mathrm{eq}}=0.0876$ (equilibrium glycerol vapor), $S_{\mathrm{B}}=0.9 S_{\mathrm{B}, \mathrm{eq}}$ and $S_{\mathrm{B}}=0.8 S_{\mathrm{B} \text {,eq }}$ assuming a $7 \mu \mathrm{m}$ diameter microdroplet. The microdroplet is assumed to be exposed to the infrared laser for $8 \mathrm{~s}$ at each laser power. 


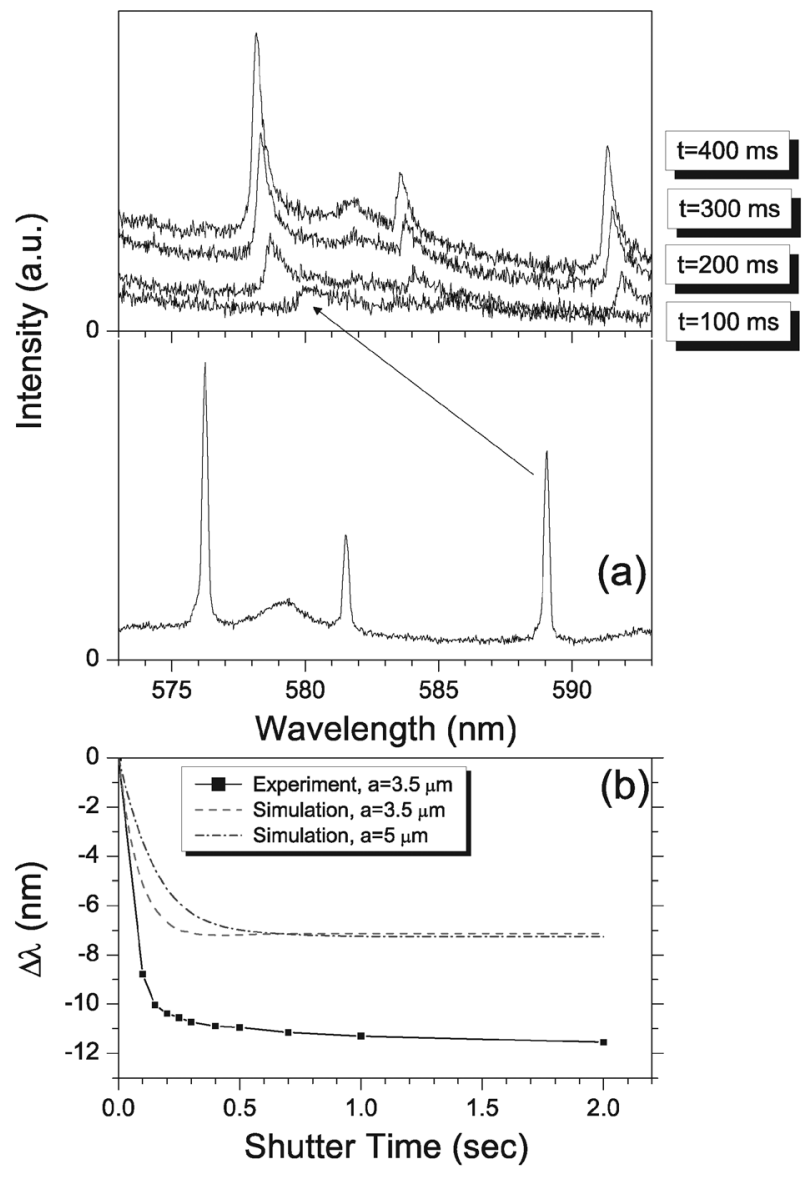

Fig. 9 Transient analysis of spectral tuning under relative water humidity $S_{\mathrm{A}}=0.8434$. (a) Fluorescence spectra recorded for a $7.0 \mu \mathrm{m}$ diameter microdroplet with no infrared laser excitation (lower plot) and upon exposure of the microdroplet to $P_{\text {inc }}=3.66 \mathrm{~mW}$ infrared laser using 100, 200, 300 and $400 \mathrm{~ms}$ shutter times (upper plot). (b) Solid line denotes the spectral shifts observed in (a) as a function of the shutter time. Dashed and dotted lines show the computational results considering $P_{\text {inc }}=3.66 \mathrm{~mW}$ for 7.0 and $10.0 \mu \mathrm{m}$ diameter microdroplets respectively.

performed for a $7.0 \mu \mathrm{m}$ diameter microdroplet with the incident laser power of $P_{\text {inc }}=3.66 \mathrm{~mW}$ are shown in Fig. 9 together with the model predictions. Simulations are performed for $7.0 \mu \mathrm{m}$ as well as $10.0 \mu \mathrm{m}$ diameter microdroplets in order to demonstrate the effects of microdroplet size on the time response. As can be seen in the top plot of Fig. 9a, the WGMs are observed to gradually drift toward their stable positions with increasing shutter time. Upon exposure to the infrared laser, microdroplet size reduces quickly mainly due to evaporation of water content and then a nearly steady state is reached. In Fig. 9b we plot the spectral position at the maximum intensity point of a WGM as a function of the shutter time together with the computational results. In this Figure, the measured and calculated maximum spectral drifts are in qualitative agreement, consistent with the results presented in Fig. 6a. In contrast, in Fig. 9b a good agreement is seen between the measured and calculated transient times. For a $7.0 \mu \mathrm{m}$ diameter microdroplet, both experimental and computational results reveal the time elapsed for the WGM to drift by $90 \%$ of its total drift to be approximately $\sim 200 \mathrm{~ms}$.

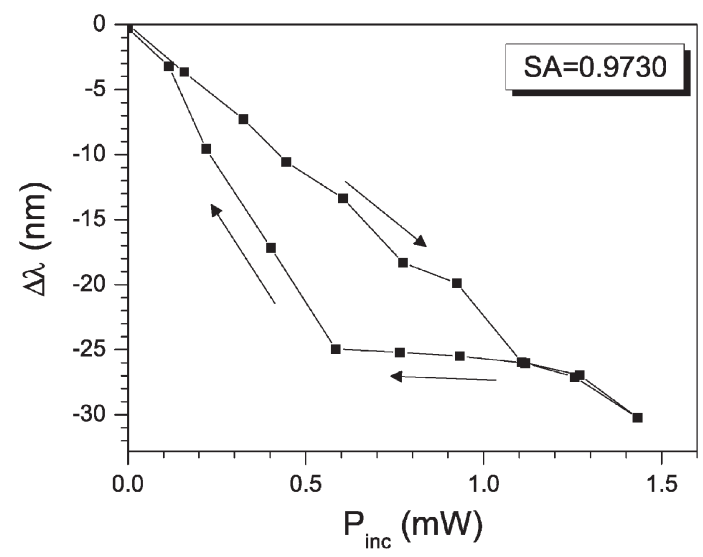

Fig. 10 Hysteresis in spectral drifts $(\Delta \lambda)$ observed for an $11.1 \mu \mathrm{m}$ diameter microdroplet. A nearly linear relationship is observed between $\Delta \lambda$ and $P_{\text {inc }}$ as $P_{\text {inc }}$ is increased from 0 to $1.43 \mathrm{~mW}$ while a significant deviation from this linear relationship is observed at about $P_{\text {inc }}=1.11 \mathrm{~mW}$ as $P_{\text {inc }}$ is reduced back to zero.

For a $10.0 \mu \mathrm{m}$ diameter microdroplet, simulations indicate that this elapsed time increases to $\sim 350 \mathrm{~ms}$. We should note that for all cases plotted in Fig. 9a the full steady state is not reached and the microdroplet size continues to decrease after this initial period of time but with a much slower rate due to the evaporation of glycerol. Although not presented here, simulations show that transient response deteriorates with increasing relative water humidity. For $7.0 \mu \mathrm{m}$ diameter microdroplet, the time elapsed for the WGMs to drift by $90 \%$ of their total drift $(\sim-7 \mathrm{~nm})$ is found to be 0.1 , 0.2 and $1.8 \mathrm{~s}$ for $S_{\mathrm{A}}=0.7530,0.8434$ and 0.9730 respectively.

For some microdroplets, especially with relatively large diameters ( $<5 \%$ of the total microdroplets studied), hysteresis is observed in the spectral drifts when the infrared laser power is varied. Such an exemplary hysteresis curve is plotted in Fig. 10 for an $11.1 \mu \mathrm{m}$ diameter microdroplet. This behavior, which is in contrast to our theoretical model, is attributed to the WGM resonances observed in laser absorption. The presence of the superhydrophobic surface will result in the deviation of the focal spot at the microdroplet center from an ideal Gaussian. This may lead to the observation of the WGM resonances in laser absorption plotted in Fig. 3. Such a high quality absorption will give rise to spectral positions at which the spectral drift of the WGMs will almost stop as the laser power is decreased from a maximum value towards 0 . Using the WGM absorption resonances we have recently demonstrated a volume stabilization mechanism when the laser illuminates the microdroplet in the vicinity of the rim. ${ }^{29}$

\section{Conclusions}

Spectral tuning of a water-glycerol microdroplet by local heating with a focused infrared laser beam has been studied experimentally by optical spectroscopy and computationally using a lumped system formulation for the mass and heat transfer between the microdroplet and the chamber. The tuning mechanism is based on the size change of the binary microdroplet controlled by focused infrared laser through evaporation and condensation of contents of the microdroplet 
due to laser-induced heating. The theoretical results are found to be in good qualitative agreement with the experimental measurements. The effects of chamber humidity, size of microdroplet and laser power on the tuning mechanism are examined. It is found that highly reversible large $(>30 \mathrm{~nm}$ ) spectral tuning can be achieved using this technique. From the experimental and theoretical results, we draw the following conclusions.

1. The spectral drift caused by the deformation due to the optical scattering force is shown to be much smaller than the spectral resolution of the experimental setup for the range of laser powers used in the experiments. Therefore the optical scattering force cannot be the main mechanism for the large spectral tuning observed experimentally by Kiraz et al. ${ }^{8}$

2. Observed large size changes in microdroplets are mainly caused by evaporation of their water content in response to the laser-induced heating.

3. Spectral tuning is essentially independent of the microdroplet size but is very sensitive to the relative water humidity in the chamber. The sensitivity of tuning increases rapidly with increasing relative water humidity.

4. Although the volatility of glycerol is much smaller than that of water, the evaporation of glycerol is still important for reversibility of tuning mechanism. Both computational and experimental results indicate that irreversibility is mainly caused by evaporation of glycerol and reversibility can be increased dramatically when air in the chamber is saturated with glycerol vapor.

5. The reversibility is also greatly affected by the relative water humidity and it increases rapidly with increasing the relative water humidity in the chamber.

6. The time response of the tuning mechanism is mainly determined by the size of the microdroplet and relative water humidity, and deteriorates with an increase in both of these factors. Transient time is found to be $0.1-2 \mathrm{~s}$ for the experimental parameters.

7. Some hysteresis effects are also observed especially for large microdroplets (diameter $\sim 11 \mu \mathrm{m}$ ), i.e., in about $5 \%$ of microdroplets studied in this paper. This is attributed to the WGM resonances in laser absorption.

The large spectral tuning technique presented in this article can inspire novel, largely tunable liquid microdroplet-based components for optical communication systems. The presented technique can also be used in fundamental studies in cavity quantum elctrodynamics, and in characterizing liquid aerosols on a surface. The results show that one component of the microdroplet must be ideally nonvolatile in order to make the present tuning technique fully reversible. For future studies, an inorganic salt with a good solubility can be a good substitute for glycerol. ${ }^{6}$

\section{Acknowledgements}

This work is partially supported by the Scientific and Technological Research Council of Turkey (Grant No. TÜBİTAK-105T500). The authors thank the Alexander von Humboldt Foundation for equipment donation. A. Kiraz acknowledges the financial support of the Turkish Academy of Sciences in the framework of the Young Scientist Award program (Grant No. A.K/TÜBA-GEBIP/2006-19).

\section{References}

1 K. J. Vahala, Nature, 2003, 424, 839.

2 A. Kiraz, A. Kurt, M. A. Dündar and A. L. Demirel, Appl. Phys. Lett., 2006, 89, 081118.

3 H. Tu and A. K. Ray, Chem. Eng. Commun., 2005, 192, 474- 498.

4 A. K. Ray, R. D. Johnson and A. Souyri, Langmuir, 1989, 5, $133-140$.

$5 \mathrm{H} . \mathrm{Tu}$, Application of Light Scattering in Studies of Transport, Thermodynamics, Light Absorption, Electric Properties of Single Droplets, PhD thesis, University of Kentucky, 2001.

6 J. P. Reid, H. Meresman, L. Mitchem and R. Symes, Int. Rev. Phys. Chem., 2007, 26, 139-192.

7 R. J. Hopkins, C. R. Howle and J. P. Reid, Phys. Chem. Chem. Phys., 2006, 8, 2879-2888.

8 A. Kiraz, S. Ç. Yavuz, Y. Karadağ, A. Kurt, A. Sennaroglu and H. Cankaya, Appl. Phys. Lett., 2007, 91, 231102.

9 A. Kiraz, S. Ç. Yavuz, Y. Karadağ, A. Kurt, A. Sennaroglu and H. Çankaya, Appl. Phys. Lett., 2008, 92, 129902.

10 M. H. Fields, J. Popp and R. K. Chang, in Progress in Optics, ed. E. Wolf, Elsevier, New York, 2000, vol. 41, pp. 1-95.

11 E. J. Davis, Aerosol Sci. Technol., 1997, 26, 212-254.

12 A. Ashkin and J. M. Dziedzic, Science, 1975, 187, 1073.

13 A. Ashkin and J. M. Dziedzic, Phys. Rev. Lett., 1977, 38, 1351.

14 S. Arnold and L. M. Folan, Rev. Sci. Instrum., 1986, 57, 2250.

15 R. J. Hopkins, L. Mitchem, A. D. Ward and J. P. Reid, Phys. Chem. Chem. Phys., 2004, 6, 4924.

16 A. Ashkin, Biophys. J., 1992, 61, 569-582.

17 S.-X. Qian, J. B. Snow, H. M. Tzeng and R. K. Chang, Science, 1986, 231, 486.

18 V. V. Datsyuk, J. Mol. Liq., 2001, 84, 1308-1316.

19 G. Grehan, B. Maheu and G. Gouesbet, Appl. Opt., 1986, 25, 3539-3548.

20 J. A. Lock, Appl. Opt., 1995, 34, 559-570.

21 J. A. Lock, J. Opt. Soc. Am. A, 1998, 15, 2986-2994.

22 R. G. Picknett and R. J. Bexon, J. Colloid Interface Sci., 1977, 79, 667-677.

23 G. McHale, S. Aqil, N. J. Shirtcliffe, M. I. Newton and H. Y. Erbil, Langmuir, 2005, 21, 11053-11060.

24 MATLAB R2006b ${ }^{\circledR}$, The MathWorks, Inc., Natick, MA, USA.

25 E. J. G. Peterman, F. Gittes and C. F. Schmidt, Biophys. J., 2003, 84, 1308-1316.

26 D. K. Cheng, Field and Wave Electromagnetics, Addison-Wesley, Reading, MA, USA, 1991.

27 D. R. Lide, CRC Handbook of Chemistry and Physics, Internet Version 2005, CRC Press, Boca Ration, FL, USA, 2005, see http://www.hbcnetbase.com.

28 A. Kiraz, Y. Karadağ and A. F. Coskun, Appl. Phys. Lett., 2008, 92, 191104.

29 A. Kiraz, A. Kurt, M. A. Dündar, M. Y. Yüce and A. L. Demirel, J. Opt. Soc. Am. B, 2007, 24, 1824-1828. 